\begin{tabular}{|l|l|c|c|}
\hline Eiszeitalter u. Gegenwart & $\mathbf{4 4}$ & $\begin{array}{c}106-113 \\
2 \mathrm{Abb} .\end{array}$ & Hannover 1994 \\
\hline
\end{tabular}

\title{
Weser und Leine am Berglandrand zur Ober- und Mittelterrassen-Zeit
}

\author{
Peter RohDE*) \\ Pleistocene, Upper Terrace/Oberterrasse, Middle Terrace/Mittelterrasse, Weser River, Leine River, MAARLEVELD \\ boundary, fluvial history, mapping, gravel provenance analyses. \\ Upland border region Hannover - Osnabrück, Niedersachsen, Nordrhein-Westfalen.
}

\begin{abstract}
Kurzfassung: Bis zum Vereisungsbeginn der Elster-Kaltzeit folgte die Weser ihrem "angestammten" pleistozänen Lauf: Hameln - Springe - Nordstemmen - Ha n nover - Mellendorf - (?)Nienburg. Bei Nordstemmen nahm sie die Leine auf. Reste ihrer Obert erras s en-Kiese ermöglichen diese Rekonstruktion. Erst seit der Mit telt er ras sen-Zeit (Älterer Teil der Saale-Kaltzeit, evtl. von einem jüngeren Teil der Elster-Kaltzeit an) verläuft die Weser in dem Talabschnitt Hameln-Porta Westfalica. Von hier aus hat sie - in einer Breite bis $25 \mathrm{~km}$ - Sedimentstränge nördlich parallel zum Wiehengebirge aufgebaut. Etwa von Hunteburg westlich des Stemmweder Berges verlief sie zumindest teilweise weiter in Richtung Damme - Vechta.

Die Leine der Mittelter rassen-Zeit läßt sich von Nordstemmen über Pattensen, Gehrden, Wunstorf, Rehburg verfolgen; sie mündete etwa im Gebiet von Uchte in die Weser. Die in einer Karte zusammengefaßte flußgeschichtliche Darstellung beruht weitgehend auf Geländearbeiten der Geologischen Landesaufnahme des Niedersächsischen Landesamtes für Bodenforschung.
\end{abstract}

\section{[The Weser and Leine Rivers near the northern edge of the Niedersachsen Upland during Upper Terrace and Middle Terrace periods]}

\begin{abstract}
Up to the beginning of the Elsterian glaciation, the Weser River had quite a different course near the northern edge of the Niedersachsen Upland, than it has today. Its previous course is marked by the towns of Hameln, Springe, Nordstemmen, Hannover, Mellendorf and (?) Nienburg. Near Nordstemmen it was joined by the Leine River. The reconstruction is based on remains of the Upper Terrace/Oberterrasse gravel deposits, which contain pebbles of Thüringer Wald volcanics. Only since the Middle Terrace/Mittelterrasse period (older phase of the Saalian age and perhaps including younger phase of the Elsterian age) has the Weser flowed along the stretch between Hameln and Porta Westfalica. Downstream of the Porta gap, the river laid down alluvial deposits with a maximum lateral spread of $25 \mathrm{~km}$; these deposits border the Wiehengebirge on the north, extending westwards for about $50 \mathrm{~km}$, and then at least part of them turn north-northeastwards near Hunteburg towards Vechta. The Leine River, today a tributary of the Aller River, in the

\footnotetext{
*) Anschrift des Verfassers: Dr. P. RoHDE, Niedersächsisches Landesamt für Bodenforschung, Postfach 510153, D-30631 Hannover.
}

Middle Terrace/Mittelterrasse period followed a course marked by the towns of Nordstemmen, Pattensen, Gehrden, Wunstorf and Rehburg. It is likely to have flowed into the Weser east of Uchte.

The above fluvial history of the Weser and Leine is mainly based on mapping by the Geological Survey of Niedersachsen (Lower Saxony) and on gravel provenance analyses (6.3-12.5 $\mathrm{mm}$ fraction).

\section{Kartierung von Ober- und Mittelterrasse durch die geologische Landesaufnahme}

Bezüglich der Flußgeschichte der eiszeitlichen Weser und Leine ist der Rand bzw. das Vorland der Berglandregion Hannover - Osnabrück ein Schlüsselgebiet. Die Geologische Landesaufnahme des Niedersächsischen Landesamtes für Bodenforschung, Hannover, hat hier in den letzten 20 Jahren großflächig gearbeitet und dabei die Verbreitung der älteren Flußablagerungen auch im tieferen Untergrund erfaßt (GK 25). Daraus und aus zusätzlichen Angaben in der Literatur wird im folgenden eine Flußgeschichte der oberund der mittelterrassen-zeitlichen Weser und Leine entworfen (Abb.l) - als Beitrag zu einem Thema mit etwa 80jähriger Tradition (z. B. KurTz 1915, 1928).

Der Begriff Oberterrasse gilt für die Flußablagerungen vor dem elster-zeitlichen Eisvorstoß; er umfaßt hauptsächlich einen ersten Abschnitt der Elster-Kaltzeit und vielleicht auch einen späteren Abschnitt der vorhergehenden Kaltzeit unter Einschluß der Warmzeit zwischen beiden (?Rhume-Warmzeit); ältere $\mathrm{Ab}$ lagerungen sind mit dem Begriff im engeren Sinne nicht gemeint. Der Begriff Mittelterrasse gilt entsprechend für einen ersten Abschnitt der Saale-Kaltzeit vor dem drenthe-stadialen Eisvorstoß; nach gängigen Vorstellungen schließst er einen späteren Abschnitt der Elster-Kaltzeit und die Holstein-Warmzeit ein.

Die Kartierung der "Terrassen" - gemeint sind die Sedimentkörper - beruht i. a. auf Bohrungen, die das Festgestein erreicht haben und es erlauben, die Lage der Terrassen-Basis zu NN zu ermitteln. Niveau und 
Gefälle einer Basisfläche müssen den fluviatilen Bedingungen entsprechen - mit diesen Kriterien, die in Subrosionsgebieten und glaziären Stauchzonen selbstverständlich nicht gelten, lassen sich Kartierergebnisse überprüfen.

\section{Geröllanalysen nach Ausgangsgesteinen}

Eine wesentliche Kartierhilfe ist die Geröllanalyse nach Ausgangsgesteinen (i. a. 300-400 Stück der Fraktion 6,3-12,5 mm). Weser-Kies hat hohe Anteile an Buntsandstein und an Thüringer-Wald-Vulkaniten, dazu Kieselschiefer, Grauwacke, Gangquarz, und erscheint deshalb rotbrau n - bunt. Leine-Kies enthält demgegenüber Flammenmergel, weniger Buntsandstein, faktisch keine Vulkanite, aber viel Kieselschiefer und Grauwacke und etwas Gangquarz; er erscheint eher g ra u-bunt. Oberterrassen-Kies enthält kein nordisches Material, Mittelterrassen-Kies bis zu 5\%, selten $8 \%$ nordisches Material. Höhere Werte weisen auf fluviatil-glazifluviatile Mischsedimente oder auf glazifluviatile Umlagerung. Stark abweichend von den genannten Geröllspektren ist ein dritter Typ durch einen sehr hohen Anteil an Gang-/Milchquarz von max. 30 bis $55 \%$ gekennzeichnet; die meist gebleichten mesozoischen Sandsteine verlieren sich zwischen älteren, z. B. paläozoischen Sandsteinen sowie Quarzit, Grauwacke, Kieselschiefer und Kristallin. Der Kies erscheint w e iß -bunt. Seltene Granulit-Gerölle weisen ihn als sächsisch aus. Wo er frei von nordischem Material vorkommt, kann er einem oberterrassen-zeitlichen oder älteren Elbe-Lauf zugeordnet werden (L.Eissmann 2.4.90, L.Wolf 11.7.91, jeweils pers. Mitt.; dagegen bezog Genieser 1970 solchen Kies seines Typs "Dudenbostel-Rodenbostel" auf das Flußsystem der Saale und Mulde).

\section{Die Weser zur Oberterrassen-Zeit}

In der Flußgeschichte des hannoverschen Berglandes bewirkt der erste, d. h. elster-zeitliche Vorstoß des skandinavischen Eises eine tiefgreifende Veränderung. Bis zu dieser Zeit trat die Weser, größter Fluß zwischen Elbe und Rhein, bei Hannover aus dem Bergland. Mit geologischen Augen gesehen ist das nicht allzu verblüffend, sondern eher einleuchtend, öffnet sich doch das Bergland bereits südlich von Hannover trichterförmig in einer bis zu $10 \mathrm{~km}$ breiten fluviatilen Verebnung. Auch wenn die Saale-Kaltzeit deutlich Anteil an der Gestaltung hat, sind für eine solche Landschaftsformung mehr als eine Kaltzeit, also mehr als ein Jahrhunderttausend erforderlich.

Bereits südlich von Hameln nahm die OberterrassenWeser an der heutigen Humme-Mündung bei Klein Berkel (GK 25, Bl. 3922) Kurs nach Nordosten auf Hameln-Rohrsen (GK 25, B1. 3822; Kies auf Buntsandstein beim BHW-Verwaltungsgebäude) und durch das heutige Hamel-Tal auf Bad Münder und die Deister-Pforte (GK 25, Bl. 3723). Dort wendete sie sich nach Osten, gab dem weiten alten Prallhang nördlich Springe "letzten Schliff" und floß im heutigen HallerTal in Ostsüdost-Richtung nach Nordstemmen (GK 25, Bl. 3824), wo sie die Leine mit ihren vielen Pläner- und Flammenmergel-Geröllen aufnahm. Ihr folgender Abschnitt war durch das heutige Leine-Tal über Pattensen, Rethen (beide GK 25, Bl. 3724) und Hannover-Bemerode (GK 25, Bl. 3624) nach Norden gerichtet und verlief weiter durch das auffällig breite heutige Wietze-Tal nach Mellendorf (GK 25, Bl. 3424) und zum Brelinger Berg (ROHDE 1983, 1986, RÖHM 1990). Spätestens hier war das Flachland mit tiefer liegender Festgesteinsoberfläche erreicht. Die Richtung der Weser war von hier an wohl durch eine Senke in Westnordwest-Richtung etwa auf Nienburg zu vorgezeichnet.

Auf diesem Abschnitt war bei Rodenbostel (GK 25, Bl. 3423 Otternhagen, s. auch Genieser 1970) reiner Elbe-Kies glaziär verschuppt in einer Kiesgrube erschlossen; bei Hagen (GK 25, Bl. 3422 Neustadt a. R.) erbohrter Kies ist m. E. reiner Weser-Kies: Weser und Elbe müssen also auf beträchtliche Erstreckung getrennt geflossen sein, sofern die Ablagerungen der Elbe nicht zu einer kaum unterscheidbaren älteren Terrasse gehören. Erst westlich von Nienburg haben sich die beiden Flüsse wahrscheinlich vereinigt. Die ab hier folgenden, bislang seltenen Beobachtungspunkte deuten auf einen südwestlichen Flußverlauf. Nach Wolf \& SchuberT (1992) wäre der im Gebiet Celle - Nienburg zu vermutende Elbe-Lauf allerdings nicht oberterrassen-zeitlich, sondern älter und damit keine Fortsetzung ihres "Streumener" Elbe-Laufs.

Nördlich des generell ost-west-gerichteten Abschnittes der Oberterrassen-Weser und -Elbe sind im glaziär geprägten Bereich, d. h. außerhalb der Niederterrassen-Körper, Gerölle aus dem Einzugsgebiet der von Süden bzw. Osten kommenden Flüsse bisher nicht beobachtet worden. Der Sachverhalt wird seit längerem durch den Begriff der "MAARLEVELD'schen Linie" beschrieben (z.B. LüTtTg 1961 bezügl. MAARLEVELD 1954, LÜTTIG \& MEYER 1980, GüK 500 Q); ein stratigraphischer Bezug ist darin nur insofern enthalten, als Ober- und Mittelterrasse erörtert worden sind.

\section{Die Höhenlage der Oberterrasse der Weser}

Über das Basis-Niveau der Oberterrassen-Sedimente lassen sich nur für den Abschnitt Hameln - Hannover Angaben machen: es senkt sich auf ca. 60 km Erstreckung von knapp $80 \mathrm{~m}$ auf etwa $65 \mathrm{~m}$ über NN (Rohde 1983). Weiter im Norden ist die Lagerung glaziär gestört.

Das Niveau der Oberterrassen-Vorkommen zwischen Hameln und Hannover schließt einen Verlauf der da- 


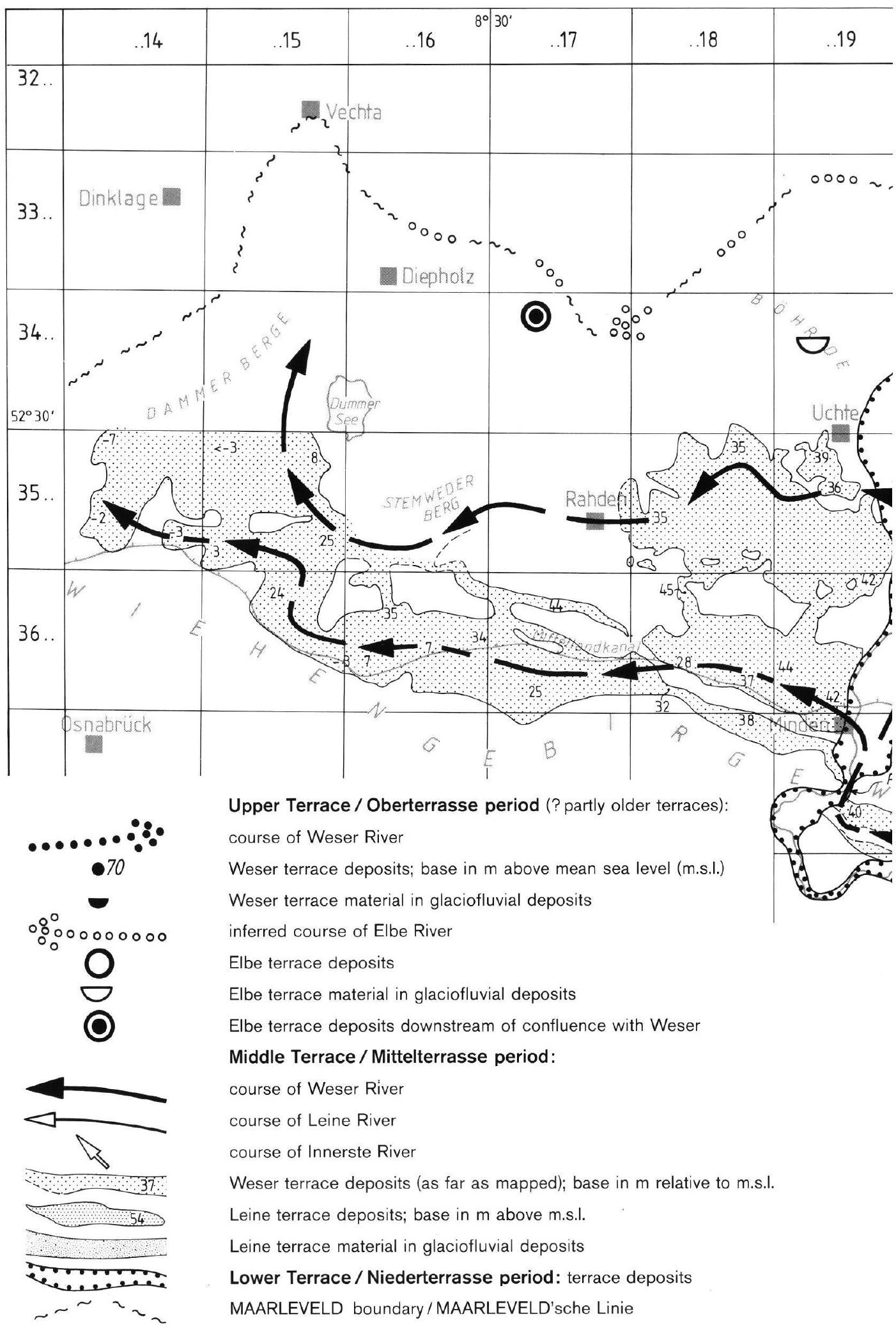




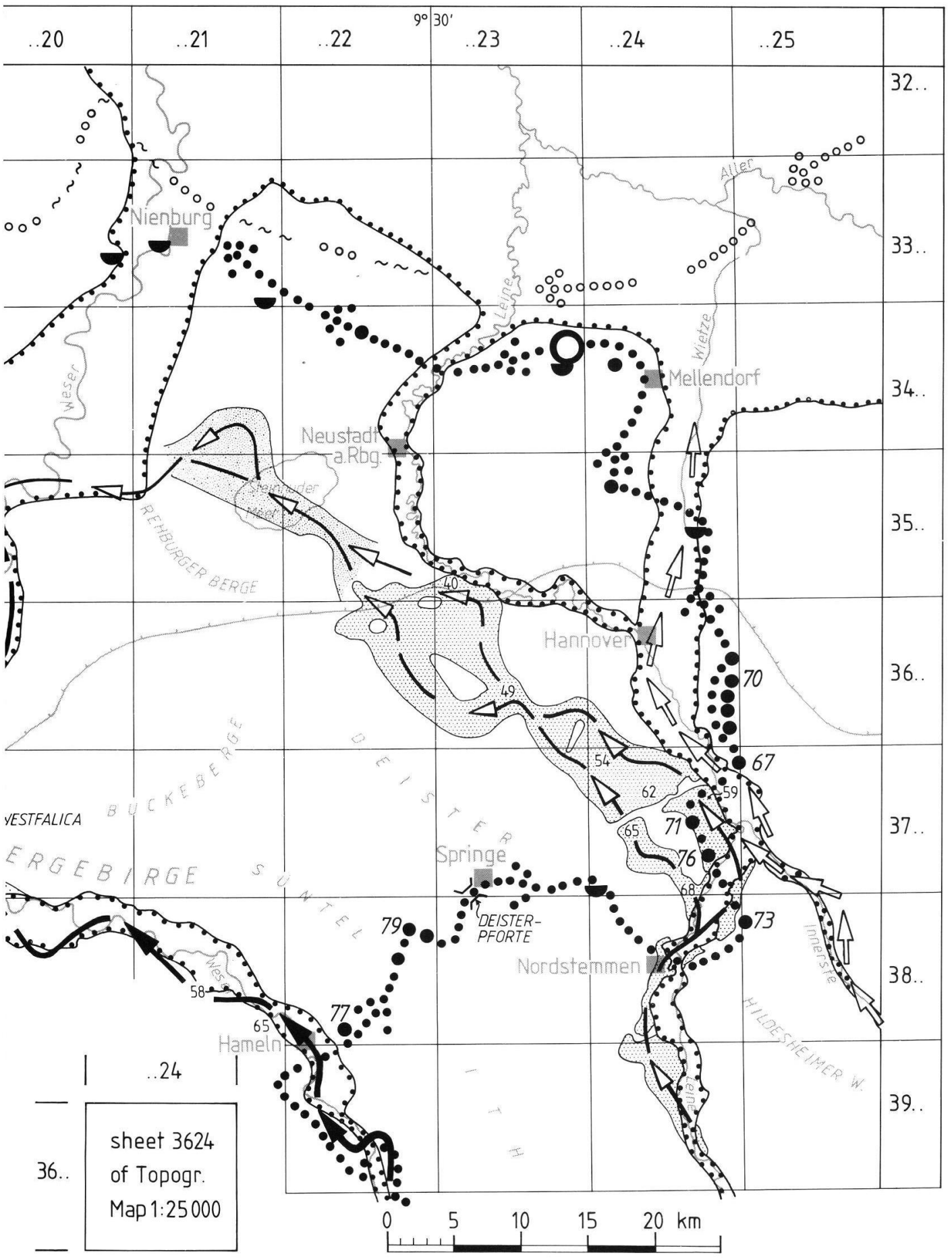

Abb.1: Die eiszeitlichen Flüsse Innerste, Leine, Weser und Elbe im Bergland-Randgebiet Hannover - Osnabrüick. Maßstab 1:500.000.

Entwurf: P. ROHDE, Dez. 1993, nach Ergebnissen der Geologischen Landesaufnahme von Niedersachsen, Nordrhein-Westfalen und Preußen. Ausführung: H. MLingeling. 
maligen Weser durch die Senke zwischen Deister und Süntel (LüTTIG 1960), d. h. von Bad Münder über Lauenau nach Rodenberg, aus, da hier die Festgesteinsoberfläche an ihren tiefsten Stellen auch heute noch dieses Niveau überragt.

Im Stadtgebiet von Hannover ist das genannte BasisNiveau noch vor der elster-zeitlichen Eisbedeckung unterschnitten worden, bei Hannover-Bemerode bis auf $39 \mathrm{~m}$ üb. NN, also um etwa $30 \mathrm{~m}$ (Abb. 2 sowie ROHDE 1992). In der Füllung der Rinne wurde basal reiner Weser-Kies, verbreitet aber Mischkies mit bis zu 5\% skandinavisch-baltischer Gerölle (Fraktion 6,3$12,5 \mathrm{~mm}$ ) erbohrt. Der Kies in der Rinne geht nach Süden auf ca. $10 \mathrm{~km}$ Entfernung in Schmelzwasser-Kies über; er wird örtlich von Mittelterrassen-Kies der Leine bedeckt und ist daher eindeutig elster-zeitlich. Die Lage unter der Mittelterrasse unterscheidet die Rinnenfüllung selbst bei gleichem Geröllspektrum deutlich vom Oberterrassen-Körper, dessen Basis 6 - $8 \mathrm{~m}$ höher als die der Mittelterrasse liegt. Die Rinnenbildung muß in einer einleitenden Phase des glaziären Geschehens der Elster-Kaltzeit stattgefunden haben; sie hier einem zweiten Vorstoß zuzuordnen (vgl. Wolf \& SCHUberT 1992), wäre nur unter komplizierten zusätzlichen Annahmen möglich.

\section{Die Weser zur Mittelterrassen-Zeit}

Durch die erste Eisbedeckung wurde die Landschaft wesentlich umgestaltet. Die Gletscher drangen ins Bergland ein und erreichten etwa die Tallinie Bodenwerder - Hameln; südwestlich der Linie Hameln Rinteln - Porta Westfalica stießen sie wohl noch ins Lippische Bergland vor (z.B. KALTWANG 1992, z.B. GÜK 500 Q). Für die Weser war der Weg von Hameln über Springe und Hannover damit verspert. Sie benutzt seither das ehemalige Nebental zwischen Wesergebirge und Lippischem Keuperbergland. Die hier zu erwartenden fluviatil-glaziären Mischbildungen scheinen vollständig wieder ausgeräumt worden zu sein. Erst in den Mittelterrassen-Ablagerungen gibt sich die Weser wieder zu erkennen. Die Vorkommen ziehen sich von Gut Helpensen (GK 25, Bl. 3821) westlich von Hameln bis etwa Rinteln (GK 25, Bl. 3820) am linken Talrand entlang und liegen im Abschnitt Veltheim Hausberge (GK 25, Bl. 3719) unter den drenthe-stadialen Schmelzwasser-Sedimenten der "Hausberger Schweiz". Austrittstelle der Weser aus dem Bergland ist von nun an das Nadelöhr Porta Westfalica, eine erdgeschichtliche "Behelfslösung" im Vergleich zu der Talweitung südlich von Hannover.

Nördlich des Wiehengebirges haben Subrosionssenken über Münder-Mergel-Salinar sowie der elster-zeitliche Schmelzwasser-Abfluß direkt nach Westnordwesten den weiteren Weg vorgezeichnet. Auffällig ist hier die gewaltige Quererstreckung der Mittelterras-
sen-Vorkommen von Minden bis Uchte (ca. $25 \mathrm{~km}$; WORTMANn 1968 sowie GK 25, B1. 3519). Nach rd. $50 \mathrm{~km}$ Verlauf nördlich des Wiehengebirges bis an den Kalkrieser Berg setzt sich die Mittelterrasse unter jüngeren Schichten z. T. nach Nordnordost fort (MeNGELING 1986, 1993) und läßt sich im Stauchungsgebiet der Dammer Berge bis Vechta (TK 25, Bl. 3215) verfolgen. Wie Weser-Material im Stauchungsgebiet der Fürstenauer Berge zeigt, ist der weitere Verlauf der Weser zur Mittelterrassen-Zeit in westlicher Richtung zu suchen.

\section{Die Höhenlage der Mittelterrasse der Weser}

Die Basis des Mittelterrassenkörpers liegt bei Hameln randlich $65 \mathrm{~m}$ über NN (KulLe 1985), hat im Gebiet Hausberge - Porta Westfalica ihre tiefsten Werte bei ca. 40 m über NN (RöHm 1985) und reicht im Nordteil des Blattgebietes 3518 Diepenau der GK 25 bei Essern bis etwa $35 \mathrm{~m}$ über NN hinab. Der hier verlaufende Strang mit tiefliegender Basis hat - südlich eines Zwischenbereiches - eine Entsprechung am Fuß des Wiehengebirges, wo allerdings Absenkungen durch Subrosion über Salzgesteinen des Münder Mergel sich ausgewirkt haben können. Im höher gelegenen Zwischenbereich könnte die heutige Basislage durch glaziäre Stauchung beeinflußt sein (WORTMANN 1971). Erwähnt sei hier ein Problem, das das Gebiet Veltheim - Hausberge (GK 25, Bl. 3719) betrifft: eine übertiefte, m. E. glaziäre ehemalige Rinne, die bei Holzhausen bis $19 \mathrm{~m}$ über NN hinabreicht (RöHм 1985). Es wird sich nur unter günstigen Bedingungen klären lassen, ob die Rinnenstruktur unter dem Mittelterrassen-Körper ansetzt (Elster-Glazifluviatil) oder diesen durchschneidet (Drenthe-Glazifluviatil).

\section{Die Leine zur Mittelterrassen-Zeit}

Die Mittelterrassen-Weser hat sich weitab von der Mittelterrassen-Leine bewegt. War deren Weg im Rahmen der Geologischen Landesaufnahme bis in das Steinhuder-Meer-Gebiet verfolgt worden (JORDAN \& Voss 1978), so läßt sich nun auch die ehemalige Verbindung zur Weser geröllanalytisch aufzeigen.

In der vom Elster-Eis geprägten Landschaft führte je ein Mittelterrassen-Strang von Schulenburg bzw. Schliekum aus der nördlichen Richtung des Leine-Tals nach Nordwesten (GK 25, Bl. 3724). Am Benther Berg und Stemmer Berg vorbei (GK 25, Bl. 3623) lassen sich die grau-bunten Kiese leicht bis in die Gegend von Gümmer (GK 25, Bl. 3523) verfolgen. Weiter im Nordwesten findet man Leine-Material nur noch mit Schmelzwasser-Material vermischt; im Übergangsbereich sind auch Verzahnungen bekannt. Vor allem die Flammenmergel-Gerölle der Leine ermöglichen es, 


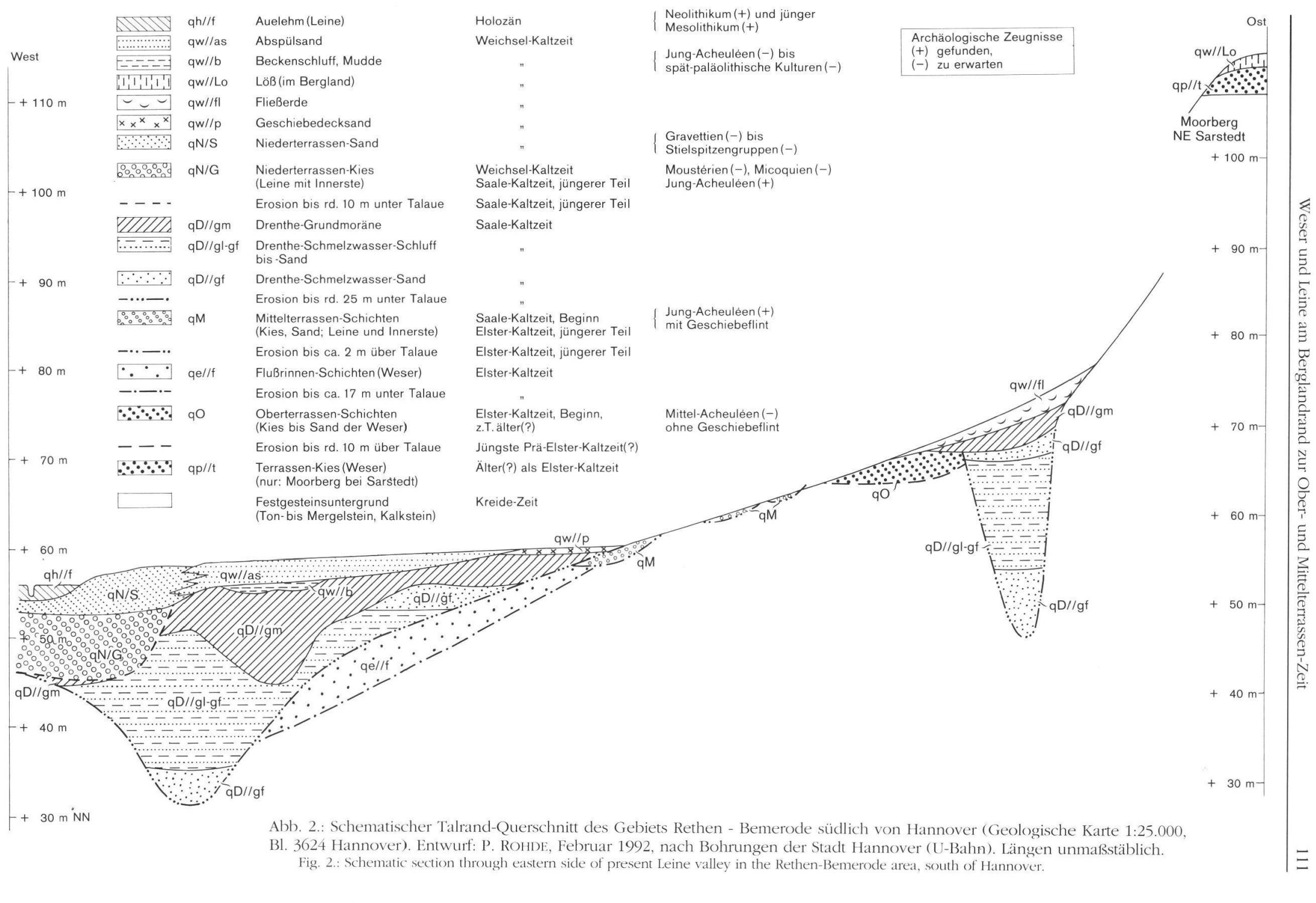


aus den Mischsedimenten den Weg der Mittelterrassen-Leine im südlichen Steinhuder-Meer-Gebiet zu rekonstruieren und gegen den etwas weiter nördlich gelegenen der Oberterrassen-Weser abzugrenzen (JorDAN \& Voss 1978). Leine-Material ist noch nordwestlich von Loccum in Schmelzwasser-Kies nachgewiesen worden (GK 25, Bl. 3520). Nur einige Kilometer weiter westlich ist die Mittelterrassen-Weser in die West-Richtung umgebogen; hier, im Gebiet von Uchte (GK 25, Bl. 3519), wird die Leine in die Weser eingemündet sein.

Die Basis des Mittelterrassen-Körpers der Leine liegt bei Schulenburg in randlicher Lage $68 \mathrm{~m}$, bei Koldingen $59 \mathrm{~m}$, bei Roloven $54 \mathrm{~m}$ über NN (GK 25, Bl. 3724), bei Ditterke 49 m (GK 25, Bl. 3623), bei Dedensen 40 über NN (GK 25, Bl. 3523). Für die Bereiche glazifluviatiler Umlagerungen ist die Terrassen-Basis nicht mehr zu ermitteln. Für den Mündungsbereich gelten etwa $37 \mathrm{~m}$ über NN (GK 25, B1. 3519).

\section{Diskussion}

Die vorliegende flußgeschichtliche Deutung schließt eine von der GK 25, Bl. 3320 Liebenau, abweichende stratigraphische Einstufung von Weser-Kiesen ein. Diese, in glaziären Stauchzonen z. T. auf Elster-Grundmoräne beschrieben, enthalten nach LÜTTIG \& MEYER (1980) in der Fraktion 20-63 mm 10-16\% bzw. 21-38\% skandinavisch-baltischer Gesteine sowie 3-11\% Milchquarz und werden von den Autoren einer Weser-Mittelterrasse nördlicher Geröllfazies zugeordnet, wobei auch Schüttungsgefüge und Sedimentfarbe berücksichtigt sind. M. E. liegt hier Mischkies mit Material der Weser-Oberterrasse und solchem der mutmaßlichen Elbe-Oberterrasse sowie mit skandinavisch-baltischem Material aus Gletscherschmelzwässern vor.

Analyse-Beispiel aus der ehemaligen Sandgrube N Bühren, GK 25, Bl. 3320 Liebenau, R: 3509890 / H: $5833410 /$ Gelände $40 \mathrm{~m}$ über NN/ Probe von 1991, 2,8-3,0 m unter Gel./ Fraktion 6,3-12,5 mm/ 390 Gerölle:

Nordische Gesteine ..............................13,8\%

Kristallin $8.2 \%$, Sandstein u. Quarzit 2,8\%, Feuerstein $2,8 \%$

Einheimisch-mesozoische Gesteine .......... 23,.3\% Buntsandstein $13.1 \%$, sonstige Sandsteine 1,3\%, Flammenmergel $0,5 \%$, sonstige Spongiengesteine $1,8 \%$, Tertiär-Quarzit u. Eisensandstein $0,5 \%$, Toneisenstein $6,1 \%$

Einheimisch-paläozoische Gesteine ...........50,3\% Thür.-Wald-Vulkanite 11,6\%, Granit 4,4\%, Glimmerschiefer u. Gneis 1,8\%, Kieselschiefer 11,0\%, Sandstein u. dunkler Quarzit 11,0\%, Grauwacke 7,2 \%, heller Quarzit 3,3\%

Quarz ............................................12,6\% Restquarz 2,6\%, Milchquarz u. Gangquarz 10,0\%.
Ob die Mischung bei der ersten oder einer zweiten Ablagerung erfolgt ist, muß offen bleiben. Gegen Mittelterrasse spricht

1. die beträchtliche nordische Komponente, denn selbst in der glaziär geprägten Umgebung z. B. von Handorf (TK 25, Bl. 3414 Holdorf), des Hohen Sühn (TK 25, B1. 3316 Diepholz) und von Vechta enthält der Mittelterrassen-Kies der Fraktion 6,3-12,5 mm nur 1 bis $4 \%$ nordisches Material (K.-D. MEYER, 1980, bestimmte in den Dammer Bergen, Gebiet des Blattes 3414 Holdorf, in der Fraktion 20-63 mm 4 bis 7\% nordische Gesteine)

2. das Vorkommen deutlich außerhalb des derzeit bekannten Verbreitungsgebietes der Weser-Mittelterrasse (statt dessen aber in der "Zielrichtung" des etwas besser bekannten Abschnittes der Weser-Oberterrasse von den Brelinger Bergen bis Hagen N Neustadt a. R., s. Abb.1).

Einem anderen Erklärungsmodell gemäß könnte der genannte Kies nach fluviatiler Unterschneidung der Mittelterrasse (östlich von Uchte durch die Weser) fluviatil bis glazifluviatil abgelagert worden sein, kurz bevor das Drenthe-Eis bis hier vorgestoßen war. Ein solcher Vorgang entspräche dem, der zuvor bezüglich der Elster-Kaltzeit für das Gebiet von Hannover beschrieben worden ist (s. Abschn. "Die Höhenlage der Oberterrasse der Weser"). Diese Deutung könnte sich auf Kartierergebnisse im Gebiet des Blattes 3520 Loccum der GK 25 stützen, wo die entsprechende Kies-Basis bei $16 \mathrm{~m}$ über NN liegt.

K.-D. Meyer hat die Darstellung in LÜTtTig \& Meyer (1980) aus seiner heutigen Sicht bestätigt. Ich danke ihm in diesem Zusammenhang für seine vielen, auch sonst geführten kollegialen fachlichen Gespräche. Die offenen Fragen lassen sich nur durch weitere Untersuchungen beantworten. Vielleicht erleichtert die flußgeschichtliche Darstellung als ein Zwischenergebnis das gezielte Ansetzen künftiger Arbeiten.

\section{Schriften- und Kartenverzeichnis}

GFNIESER,K. (1970): Üher Quarze, Amethyste und verkieselte Fossilien. - Overdruck Gronboor en Hamer, 2: 35-64, 4 Abb., 3 Tab.; Losser/Nederland.

JORDAN, H. \& VOSS, H.H. (1978): Geröllanalytische Gliederung der pleistozänen Kiessande nordwestlich von Hannover. - Eiszeitalter u. Gegenwart, 28: 10-38, 8 Abb., 3 Tah.; Öhringen/Württ.

Kaltwang, J. (1992): Die pleistozäne Vereisungsgrenze im südlichen Niedersachsen und im östlichen Westfalen. Mitt. geol. Inst. Univ. Hannover, 33: 161 S., 7 Abb., 38 Tab., 49 Kt.; Hannover.

KuRTz, E. (1915): Die Wanderungen der mittleren Elbe bis zum Harz vor dem Rand des Inlandeises, nachgewiesen an Flußgeröllen. - Z. deutsche geol. Ges., 67: 231-274, Taf. 28; Berlin.

- (1928): Die Weser im Vereisungsgebiet während der 
ersten und zweiten Eiszeit. - Z. deutsche geol. Ges., 79 (1927): 457-514, Taf. 9; Berlin.

KulLE, S. (1985): Drenthe-stadiale Staubecken-Sedimente (Pleistozän) und ihr Lagerungsverband aus zwei Aufschlüssen im Wesertal zwischen Rinteln und Hameln. Dipl.-Arb. Univ. Hannover: 82 S., 18 Abb., 8 Tab., 4 Taf., 3 Anl.; Hannover. - [Unveröff.]

LÜTTIG, G. (1960): Neue Ergebnisse quartärgeologischer Forschung im Raume Alfeld - Hameln - Elze. - Geol. Jb., 77: 337-390, 11 Abb., 5 Tab., 3 Taf.; Hannover.

- (1961): Neue Interglazialvorkommen bei Liebenau a.d. Weser. - Geol. Jb., 78: 173-197, 6 Abb., 1 Tab.; Hannover.

- \& MEYER, K.-D. (1980): Geologische Karte von Niedersachsen 1: 25 000, mit Erläuterungen, Bl. 3320 Liebenau. - 118 S., 9 Abb., 12 Tab., 7 Kt.; Hannover.

MaARleveld, G. C. (1954): Über fluviatile Kiese in Nordwestdeutschland. - Eiszeitalter u. Gegenwart, 4/5 10-17, 2 Abb.; Öhringen/Württ

Mengeuing, H. (1986): Geologische Karte von Niedersachsen 1 : 25 000, mit Erläuterungen, Bl. 3514 Vörden. 125 S., 35 Abb., 5 Tab., 7 Kt.; Hannover.

- (1993): Geologische Karte von Niedersachsen 1: 25 000, mit Erläuterungen, Bl. 3515 Hunteburg. - 189 S., 57 Abb., 11 Tab., 7 Kt,; Hannover.

Meyer, K.-D. (1980): Zur Geologie der Dammer und Fürstenauer Stauchendmoränen (Rehburger Phase des Drenthe-Stadiums). - Festschr. G. Keller: 83-104, 3 Abb.. 1 Tab., 1 Taf.; Osnabrück (Verlag A.Th.Wenner).

RÖHм, H. (1985): Bau und Zusammensetzungen Saale-Eiszeitlicher Sedimentkörper im Raum Hausberge - Veltheim/Weser. - Dipl.-Arb. u. Kartier. Univ. Hannover: 107 S., 53 Abb., 7 Anl.: Hannover. - [Unveröff.]

- (1990): Untersuchungen zur Saale-zeitlichen Tal- und Flußgeschichte in der Wedemark N Hannover. - Schwerpunktprogr. DFG "Fluviale Morphogeodynamik im Jüngeren Quartär", DFG Gr 813/3-2: 63 Seiten, 38 Abb., Tab.; Bonn-Bad Godesberg.

ROHDE, P. (1983): Geologische Karte von Niedersachsen 1: 25 000, mit Erläuterungen, Bl. 3724 Pattensen. - 192 S., 43 Abb., 31 Tab., 2 Taf., 8 Kt.; Hannover.

- (1986): Geo-Umwelt im Bergland-Quartär zwischen oberer Weser und Leine. - DeuQuA, 23. Tag., Exkurs. B: 45 S., 19 Abb., 1 Tab., 1 Beil.; Hannover.

- (1992): Leinetal südlich Hannover. - In: S. VeIL [Hrsg.]: Erläuterungen zu den Tagesexkursionen I und II der Hugo-Obermaier-Gesellschaft anläßlich ihrer Tagung im April 1992 in Hannover: 28-31, Abb. 11; Hannover (Ndrs. Landesmuseum).

WOLF, L. \& SCHUBERT, G. (1992): Die spättertiären bis elstereiszeitlichen Terrassen der Elbe und ihrer Nebenflüsse und die Gliederung der Elster-Kaltzeit in Sachsen. - Geo- profil, 4 (1992): 1-43, 18 Abb., 25 Tab.; Freiberg.

WORTMANN, H. (1968): Die morphogenetische Gliederung der Quartärbasis des Wiehengebirgsvorlandes in Nordwestdeutschland. - Eiszeitalter u. Gegenwart, 19 227-239, 4 Abb., 1 Tab., 1 Taf.; Öhringen/Württ.

- (1971): Geologische Karte von Nordrhein-Westfalen 1 : 25 000, mit Erläuterungen, Bl. 3617 Lübbecke und Bl. 3618 Hartum. - 214 S., 24 Abb., 13 Tab., 3 Taf., 2 Kt. Krefeld.

GK 25 - Geologische Karte von Niedersachsen/ NordrheinWestfalen/ Preußen 1: 25 000, mit Erläuterungen. - Hannover/Krefeld/Berlin.

Folgende Blätter:

3320 Liebenau

3321 Nienburg/W.

[LÜTTIG \& MEYER]

[VOSs]

3420 Stolzenau

[HÖFLE]

3421 Husum

3422 Neustadt a. R.

3423 Otternhagen

[Voss]

[JORDAN, H.] "

Hannover 1980

1991

1981

1982

1974

1967

1986

3514 Vörden

3515 Hunteburg

3518 Diepenau

3519 Uichte Süd

3520 Loccum

3521 Rehburg

3522 Wunstorf

3523 Garbsen

3524 Isernhagen

[LANG]

[Mengeling]

[MENGELIYG]

1993

[ROHDE, lfd. Kartierung] "

[ROHDE, Kt.-Mskr.1992] "

[ROHDE]

[IORDAI. H.]

[Voss]

[ROHDE]

[LANG]

3615 Bohmte

3616 Pr. Oldendorf

3617 Lübbecke und

3618 Hartum

3619 Petershagen

3623 Gehrden

3624 Hannover

[HINZE]

[HINZF]

3719 Minden

3724 Pattensen

3820 Rinteln

[WORTMANN]

[WORTMANY]

[EBERT \& GRUPE]

[DIETZ]

[GRLPE, KOERT \& STACH]

[ROHIE]

[NaUmany]

3821 Hess.Oldendorf

3822 Hameln

3824 Elze

[NAUmanN]

[NAUMANN \& BURRE]

[LёTTIG, kart. 1953/57.

VINKEN, kart. 1975/76]

[GRUPE]

[JORDAI, H.]

1992

1979

1979

1978

1962

1982

1983

3922 Kirchohsen

3924 Gronau

1971

1968

1928

1959

1933

1983

1922

1927

1927

Hannover

Berlin 1916

Hannover

1987

GÜK 500 Q - Quartärgeologische Übersichtskarte von Niedersachsen 1: 500 000. - 1 Kt.; Hannover (Ndrs. Landesamt f. Bodenforschung). - [In Vorbereit., Mskr. 1993]

Manuskript eingegangen am 22. 11. 1993 